\title{
AVALIAÇÃO DO NÍVEL DE ATIVIDADE FÍSICA EM INDIVÍDUOS PORTADORES DE HIPERTENSSÃO ARTERIAL SISTÊMICA DA UNIDADE DE ESTRATÉGIA DE SAÚDE DA FAMÍLIA DO MUNICÍPIO DE ACREÚNA- GO
}

\author{
Getúlio Antonio de Freitas Filho' \\ Eliene Barbosa Ferreira ${ }^{2}$ \\ Nulciene Firmino de Freitas ${ }^{3}$ \\ Leonardo Squinello Nogueira Veneziano 4 \\ Rejane Maria Cruvinel Cabral ${ }^{5}$ \\ Roberto Dias ${ }^{6}$ \\ Lya Karla Manso Miranda ${ }^{7}$ \\ Kátia da Silveira Ferreira ${ }^{8}$ \\ Renata do Nascimento Silva ${ }^{9}$ \\ Tairo Vieira Ferreira ${ }^{10}$ \\ Fernando Duarte Cabral ${ }^{11}$
}

Resumo: A hipertensão arterial sistêmica (HAS), também conhecida como pressão alta, é um mal que ataca milhões de pessoas em todo o mundo. Estudos epidemiológicos apontam que cerca de $20 \%$ da população brasileira sofre com hipertensão. Este trabalho tem por objetivo a avaliação do nível de atividade física em indivíduos portadores de hipertensão arterial sistêmica de unidade de estratégia de saúde da família do município de Acreúna-GO. Foram estudados 33 indivíduos hipertensos, sendo 10 do sexo masculino e 23 do sexo feminino, que frequentam o programa de acompanhamento de hipertensos da Estratégia de Saúde da Família (ESF) Sol Nascente. Para tanto, os indivíduos analisados responderam ao IPAQ (questionário internacional de medidas prevalentes de atividades físicas) versão curta para verificação do nível de atividade física. Conclui-se que, no geral, quer seja do sexo masculino ou feminino, ambos têm uma boa prática de atividade física. Embora não seja a ideal, a atividade física praticada, alicerçada pelo tratamento medicamentoso a eles oferecido, Ihes proporciona uma boa qualidade de vida.

Palavras-chave: Hipertensão arterial; Atividade física; IPAQ.

\footnotetext{
1 Professor(a) Faculdade de Fisioterapia/Instituto de Ensino Superior de Rio Verde, Brasil. E-mail: getulio@faculdadeobjetivo.com.br.

${ }^{2}$ Fisioterapeuta, Instituto de Ensino Superior de Rio Verde, Brasil. E-mail: eliene@faculdadeobjetivo.com.br.

3 Instituto de Ensino Superior de Rio Verde, Brasil. E-mail: nulciene@faculdadeobjetivo.com.br.

4 Instituto de Ensino Superior de Rio Verde, Brasil. E-mail: leosnv@yahoo.com.br.

${ }^{5}$ Instituto de Ensino Superior de Rio Verde, Brasil. E-mail: rejane@faculdadeobjetivo.com.br.

${ }^{6}$ Instituto de Ensino Superior de Rio Verde, Brasil. E-mail: fisiodias@uol.com.br.

7 Instituto de Ensino Superior de Rio Verde, Brasil. E-mail: Iya@faculdadeobjetivo.com.br.

8 Instituto de Ensino Superior de Rio Verde, Brasil. E-mail: katia@faculdadeobjetivo.com.br.

9 Instituto de Ensino Superior de Rio Verde, Brasil. E-mail: renata@faculdadeobjetivo.com.br.

10 Instituto de Ensino Superior de Rio Verde, Brasil. E-mail: tairo@faculdadeobjetivo.com.br.

11 Professor Orientador, Instituto de Ensino Superior de Rio Verde/Faculdade de Fisioterapia, Rio Verde-Go.
} 